\title{
Correction to: The renin-angiotensin system and specifically angiotensin-converting enzyme 2 as a potential therapeutic target in SARS-CoV-2 infections
}

\author{
Francesco Ferrara ${ }^{1} \cdot$ Antonio Vitiello $^{1}$ \\ Published online: 5 November 2021 \\ ๑) Springer-Verlag GmbH Germany, part of Springer Nature 2021
}

\author{
Correction to: Naunyn-Schmiedeberg's Archives of Pharmacology (2021) 394:1589-1593 \\ https://doi.org/10.1007/s00210-021-02108-z
}

The authors regret that in the text of the manuscript at the paragraph with title 'Pharmacological target of SARS$\mathrm{CoV}-2$ ' there are parts in non-English language. In order to meet the high standard of Naunyn-Schmiedebergs Archives of Pharmacology we would like to perform a correction that would concern only the form of the language and not the scientific content.

The authors apologize for any inconvenience caused.

\section{Pharmacological target of SARS-CoV-2}

In view of the severity of the COVID-19 pandemic, there is an urgent need for effective therapies against SARS-CoV-2. As already described, ACE-2 is the cellular entry receptor of SARS-CoV-2, and it is also a key component of the RAS. Therefore, a full understanding of the correlation between ACE-2 and SARS-CoV-2 would be of critical importance to act with targeted pharmacological intervention strategies (Ferrara and Vitiello 2020; Vitiello and Ferrara 2021d; Ferrara and Vitiello 2021b; Muslim et al. 2020). However, although ACE-2 has been identified as a receptor for the SARS-CoV-2, there may be other SARS-CoV-2 co-receptors yet to be discovered (Muslim et al. 2020). This raises

The original article can be found online at https://doi.org/10.1007/ s00210-021-02108-z.

Francesco Ferrara

francesco.ferrara@uslumbria1.it

Antonio Vitiello

antonio.vitiello2@uslumbria1.it

1 Pharmaceutical Department, Usl Umbria 1, A. Migliorati Street, 06132 Perugia, Italy additional important implications for the therapeutic targets of SARS-CoV-2 (Marovich et al. 2020; Vitiello et al. 2021d; Singh et al. 2021). One of the most important strategies to control viral infections is to block the initial binding of the virus to its functional receptors. Several candidate drugs have been developed to block binding of S protein and ACE2, including drugs based on S protein and ACE-2. Monoclonal antibodies to COVID-19 act by neutralizing the spike protein of SARS-CoV-2, which blocks the binding of the spike protein to human ACE-2 receptors, thereby preventing subsequent viral entry into human cells and virus replication (Marovich et al. 2020; Tian et al. 2020). Treatment with a soluble recombinant human form of ACE2 (rhACE2) could prove useful as a trap effect for circulating SARS-CoV-2 and decrease viral load and hinder infection (Monteil et al. 2020). Administration of recombinant soluble human ACE2 has shown good efficacy in subjects with acute respiratory distress syndrome (ARDS) (Muslim et al. 2020). From a molecular pharmacological point of view, administration of rhACE2 activates the Ang 1-7 and Ang 1-9 synthesis pathway of the RAS system (non-classical pathway) by decreasing Ang II levels with a tendency to lower the concentration of proinflammatory cytokines (Gaddam et al. 2014). Some clinical trials show excellent results when administered in combination rhACE2 and remdesivir (Monteil et al. 2021). Theoretically, administration of soluble ACE2 protein, in sufficient quantities, binding to the spike protein of SARSCoV-2, could reduce the attachment to ACE-2 at the plasma membrane. This could be used therapeutically as a way to reduce infectivity in patients treated with COVID-19. Studies in healthy volunteers have demonstrated a reduction in Ang II after administration of soluble ACE-2 (Imai et al. 2005). In contrast, direct administration of Ang (1-7) failed to demonstrate significant effects in humans (Oudit and 
Penninger 2011). In addition, preclinical studies have shown that administration of soluble ACE-2 can decrease lung injury (Haschke et al. 2013). In patients with acute respiratory distress syndrome (ARDS), hrACE2 (GSK2586881) was well-tolerated (Khan et al. 2017). Human rACE2 caused a decrease in circulating Ang II levels (73), whereas angiotensin (1-5) and angiotensin (1-7) levels increased and remained elevated for $48 \mathrm{~h}$ (Khan et al. 2017). Some in vitro and in silico assays revealed two compounds (xanthenone and resorcinolnaphthalein) that increase ACE2 activity in a dose-dependent manner ( $\mathrm{Li}$ et al. 2020b). They may be useful in increasing Ang II turnover in Ang (1-7). Finally, vitamin $\mathrm{D}$ is known for its anti-inflammatory effects. The hormonal form of vitamin $\mathrm{D}$, calcitriol $(1,25(\mathrm{OH}) 2 \mathrm{D} 3)$, is a negative endocrine regulator of the RAS and inhibits renin biosynthesis, and it blocks the expression of ACE and Ang II and elevates ACE2 levels in LPS-induced acute lung injury (Xu et al. 2015). Thus, it could potentially play a role in combating SARS-CoV-2 infection and related complications.

Publisher's note Springer Nature remains neutral with regard to jurisdictional claims in published maps and institutional affiliations. 